\title{
Finite-difference time-domain analysis of time-resolved terahertz spectroscopy
} experiments

\author{
Larsen, Casper; Cooke, David G.; Jepsen, Peter Uhd
}

Published in:

Optical Society of America. Journal B: Optical Physics

Link to article, DOI:

10.1364/JOSAB.28.001308

Publication date:

2011

Document Version

Publisher's PDF, also known as Version of record

Link back to DTU Orbit

Citation (APA):

Larsen, C., Cooke, D. G., \& Jepsen, P. U. (2011). Finite-difference time-domain analysis of time-resolved

terahertz spectroscopy experiments. Optical Society of America. Journal B: Optical Physics, 28(5), 1308-1316.

https://doi.org/10.1364/JOSAB.28.001308

\section{General rights}

Copyright and moral rights for the publications made accessible in the public portal are retained by the authors and/or other copyright owners and it is a condition of accessing publications that users recognise and abide by the legal requirements associated with these rights.

- Users may download and print one copy of any publication from the public portal for the purpose of private study or research.

- You may not further distribute the material or use it for any profit-making activity or commercial gain

- You may freely distribute the URL identifying the publication in the public portal 


\title{
Finite-difference time-domain analysis of time-resolved terahertz spectroscopy experiments
}

\author{
Casper Larsen, ${ }^{1, *}$ David G. Cooke, ${ }^{2, *}$ and Peter Uhd Jepsen ${ }^{1,3}$ \\ ${ }^{1}$ DTU Fotonik-Department of Photonics Engineering, Technical University of Denmark, 2800 Kgs., Lyngby, Denmark \\ ${ }^{2}$ Department of Physics, McGill University, Montreal, Quebec, Canada H3A 2 T8 \\ ${ }^{3}$ e-mail: puje@fotonik.dtu.dk \\ *Corresponding author: cooke@physics.mcgill.ca
}

Received February 7, 2011; accepted March 8, 2011;

posted March 21, 2011 (Doc. ID 142356); published April 28, 2011

In this paper we report on the numerical analysis of a time-resolved terahertz (THz) spectroscopy experiment using a modified finite-difference time-domain method. Using this method, we show that ultrafast carrier dynamics can be extracted with a time resolution smaller than the duration of the $\mathrm{THz}$ probe pulse and can be determined solely by the pump pulse duration. Our method is found to reproduce complicated two-dimensional transient conductivity maps exceedingly well, demonstrating the power of the time-domain numerical method for extracting ultrafast and dynamic transport parameters from time-resolved $\mathrm{THz}$ spectroscopy experiments. The numerical implementation is available online. (c) 2011 Optical Society of America

OCIS codes: $\quad 300.6495,300.6530,320.7130,000.4430,190.7110$.

\section{INTRODUCTION}

Observing carrier dynamics and nonequilibrium transport phenomena in semiconductors occurring on ultrafast time scales is a considerable research challenge. Several ultrafast optical measurement techniques based on ultrafast lasers and nonlinear interactions have been applied [1,2]. One of the newest ultrafast optical methods is time-resolved terahertz spectroscopy (TRTS). This technique probes low-energy charge carrier motion by interrogating the sample optically with terahertz $(\mathrm{THz})$ pulses, which are typically generated through difference frequency mixing and detected by phasesensitive electro-optic detection using $\chi^{(2)}$ crystals [3]. THz pulses are extremely broadband $(0.3-3 \mathrm{THz})$, sub-ps duration electromagnetic transients and therefore are ideal probes for ultrafast spectroscopy in the millielectron volt photon energy region. TRTS has been used in unique experiments such as observing the birth of an optically excited plasma [4] and peering inside the internal structure of a newly created exciton [5] . For recent reviews, see [ㅁ, 7$]$.

In a typical TRTS experiment interrogating carrier dynamics in a semiconductor, an optical pump is used to photoexcite electron-hole pairs. By propagating the $\mathrm{THz}$ probe pulse through or reflecting off the photoexcited region, perturbations in the $\mathrm{THz}$ probe pulse due to, e.g., free carrier absorption or phonon scattering can be measured. These perturbations give information about carrier momentum scattering rates, relaxation dynamics, and carrier densities.

In static $\mathrm{THz}$ time-domain spectroscopy, frequencyresolved data are retrieved by Fourier transforming the measured reference $E_{\text {ref }}(t)$ and sample $E_{\text {sam }}(t)$ waveforms so that $\mathrm{THz}$ pulse distortions in the experimental setup and detector crystal cancel when the transmission function $T(\Omega)=E_{\text {sam }}(\Omega) / E_{\text {ref }}(\Omega)$ is found. Material properties can then be extracted from $T(\Omega)$ by an appropriate model [].

It can be argued that for material responses much slower than the $\mathrm{THz}$ pulse probing event, the so-called quasistatic approximation is valid and the data analysis of a time-resolved experiment can be performed as if it is a static experiment [묘, $\underline{9}$. However, retrieving dynamic responses that occur during or faster than the time it takes for the THz probe pulse to traverse the excitation is a problem in the analysis of TRTS experiments. Several methods have been developed to address this problem and correct for pulse distortions. A two-dimensional (2D) Fourier transform method has been proposed in which the carrier response can be isolated in cases where the rise and fall times of the photocarrier response are on the same order of magnitude. If the induced modulation is small, the $\mathrm{THz}$ propagation equations can be linearized and solved analytically to yield the material properties $[\underline{8}, \underline{10}]$. Finally, a finite-difference time-domain (FDTD) model has been implemented to numerically propagate the probe pulse through the photoexcited sample [11]. The fundamental disadvantages of the FDTD approach are difficulties in solving the inverse problem and the fact that an a priori model must be chosen to describe the carrier dynamics and the complex optical response function (e.g., the optical conductivity).

Here, we report on an improved FDTD model that solves several problems with implementing FDTD to describe a TRTS experiment, by enabling high computational efficiency to permit iterative numerical data fitting as well as a very high flexibility in choice of carrier dynamics, as will be illustrated. In the following, the TRTS experiment is described and the modified FDTD method is introduced. We finish our paper with an exemplary analysis of a TRTS experiment of photoexcited GaAs using the FDTD method.

\section{CONCEPT OF THE TRTS EXPERIMENT}

By analysis of the data obtained in a TRTS experiment, subpicosecond photoexcited carrier dynamics can be extracted $[\underline{12}, 13]$. However, extracting the carrier responses that occur during the probing event requires significantly more effort than the slower responses and the reasons for this will be 
discussed in the following. The TRTS experiment involves an optical pump pulse and a $\mathrm{THz}$ probe pulse. The optical pump pulse excites the sample, and the THz pulse probes the dynamic response of the sample as a function of the time delay between the pump and the probe pulses, accomplished through the use of mechanical delay stages. In Fig. 1, the pump and probe pulses are illustrated, and times have been assigned to them. The pump-probe delay time is defined as $t_{p p}=\tau_{\text {probe }}-\tau_{\text {pump. }}$. By consecutively measuring the $\mathrm{THz}$ waveforms at increasing pump-probe delay times, a $2 \mathrm{D}$ map can be constructed that contains information about the pump-induced carrier dynamics.

However, at early pump-probe delay times $(<\mathrm{ps})$, the probe pulse and the carrier response, which is a convolution with the pump pulse, overlap in time. This has the consequence that the tailing part of the probe experiences very different modulation than the leading edge of the probe. This is well illustrated in [9]. Two problems arise from this fact. First, the usual Fourier transformation of the measured waveform no longer makes sense. Second, in the case of very fast responses $(<300 \mathrm{fs})$, this leads to generation of new frequency components in the $\mathrm{THz}$ pulse.

The first problem can be solved experimentally by using a property of the electro-optic detection scheme [ㄱ,14]. In this scheme, the electric field of the $\mathrm{THz}$ pulse is measured by delaying the gate or sampling pulse relative to the THz probe pulse. This is also illustrated in Fig. 1 , where the THz delay $t=$ $\tau_{\text {sampling }}-\tau_{\text {probe }}$ is defined.

If a pump-sampling time delay is defined as

$$
t_{p}=\tau_{\text {sampling }}-\tau_{\text {pump }}=t_{p p}-t,
$$

then it is possible to reconstruct a $2 \mathrm{D}$ map by the transformation $t_{p p}=t_{p}+t$. This effectively means that the reconstructed $\mathrm{THz}$ probe pulse has experienced the same pump delay and usual Fourier transform methods can be applied. Mathematically, the transient, induced polarization $\Delta P\left(t, t_{p}\right)$ can be described by [10]

$$
\Delta P\left(t, t_{p}\right)=\int E\left(t-t^{\prime}\right) \tilde{\chi}\left(t^{\prime}, t_{p}\right) \mathrm{d} t^{\prime},
$$

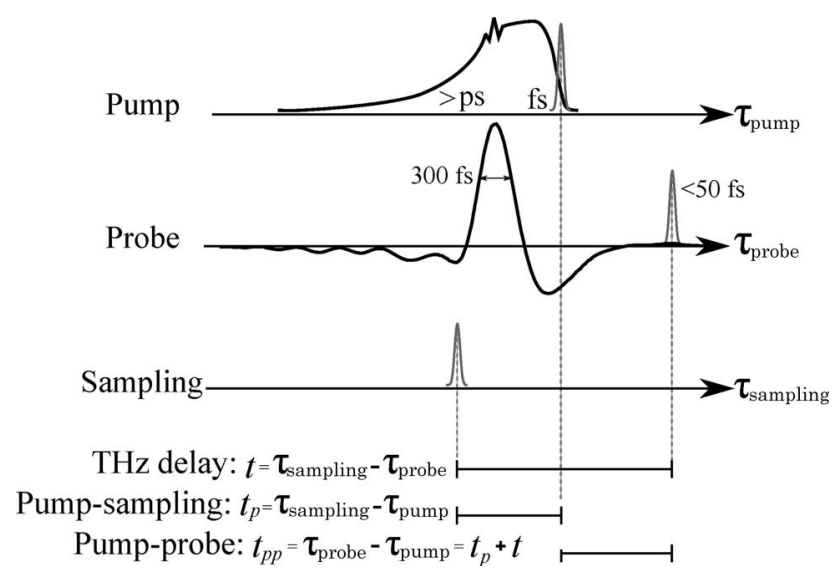

Fig. 1. Time lines of the three pulses involved in TRTS: pump, probe, and sampling pulses. Each pulse has been designated an absolute time $\tau$, and the definition of relative times $t$ are shown in the bottom. The optical pump pulse of less than 50 fs excites a carrier density in the sample that decays over time, which is illustrated at the pump time line. The THz probe pulse is shown with a full-width at half-maximum of $300 \mathrm{fs}$ of the central peak in the electric field. where $E$ is the electric field and $\tilde{\chi}$ the transient nonlinear susceptibility. In the reconstructed $2 \mathrm{D}$ map, $\Delta P$ has a parametric dependence of the pump delay time; hence, through the convolution theorem, the frequency-domain susceptibility is

$$
\chi\left(\Omega, t_{p}\right)=\Delta P\left(\Omega, t_{p}\right) / E(\Omega),
$$

where $\Omega$ is the THz frequency. Whereas the induced polarization without the reconstruction $\Delta P^{*}\left(t, t_{p p}\right)$ is given by

$$
\Delta P^{*}\left(t, t_{p p}\right)=\int E\left(t-t^{\prime}\right) \tilde{\chi}\left(t^{\prime}, t_{p p}-t\right) \mathrm{d} t^{\prime},
$$

which describes the pump and probe pulse temporal overlap and is not a convolution.

Experimentally, this reconstruction is handled in the way the mechanical delay stages are moved, i.e., the pump and sampling pulses are fixed for a given $t_{p}$ and the probe pulse is scanned [7]. Alternatively, $\Delta P^{*}\left(t, t_{p p}\right)$ can be measured by fixing the pump and probe pulses at a given $t_{p p}$ and scanning the sampling pulse followed by a numerical reconstruction. In the literature, these two methods of representing the $2 \mathrm{D}$ data have been termed modes or representations [모요.

It should be stressed that data taken in any of the representations contain the exact same information.

The second problem involves frequency mixing of the carrier response and probe pulse, and the solution requires knowledge of the $\mathrm{THz}$ pulse propagation properties in the experimental setup and detection crystal. In electro-optic detection, the THz pulse is sampled by the ultrafast sampling pulse in the detection crystal where phase mismatching and absorption will limit the detection bandwidth and efficiency. Mismatch between the group velocity of the optical pulse and the phase velocities of the $\mathrm{THz}$ frequencies leads to temporal walk-off of the pulses, which has been shown to have a lowpass-filter effect on the THz pulse [14]. Furthermore, focusing elements after the sample act as apertures for the long wavelength components; hence, it has a high-pass-filter effect. In transmission experiments, propagation in the sample after the photoexcited region must also be considered. These effects have been described analytically and calculated numerically in [15]; however, the exact beam shape is required. Alternatively, the propagation effects can be found experimentally [13]. With knowledge of these filter or response functions, distortions after the sample can be removed by deconvolution techniques $[\underline{8}, 9]$ ]. However, it is important to have in mind that these response functions act on each propagating probe pulse; hence, deconvoluting the responses from the reconstructed $2 \mathrm{D}$ map, which is synthesized by many probe pulses, does not make sense.

In the present FDTD model, both problems are overcome by the time-domain approach. It simulates the temporal overlap directly, and the response functions can be convoluted with the THz pulses so that the noise-generating deconvolutions can be avoided, as will be discussed later.

\section{FDTD MODELING OF TRTS EXPERIMENTS}

Propagation of a $\mathrm{THz}$ probe pulse through a photoexcited sample has been modeled using linearized propagation equations $[\underline{8}, \underline{10}]$ and the FDTD method [11]. The advantages of the FDTD method include no limitation of modulation 
strength and simulation of the TRTS experiment in a very direct manner where, e.g., the pumping event and carrier transport can be solved simultaneously with pulse propagation.

In [11], a TRTS experiment is simulated using FDTD; however, the model to be described in this paper has several improvements, such as the incorporation of arbitrary dispersion [16], coupled dynamics of different types of carriers, and simultaneous solving of the carrier diffusion equations. FDTD methods have been implemented in other fields $[\underline{16}, \underline{17}]$, but their implementations are not suitable for the TRTS experiment.

The principle of the FDTD method is to solve the Maxwell's equations using the finite-difference approximation of the differential operators [16]. It is popular to apply the central difference approximation (CDA) due to second-order accuracy. In modeling of the TRTS experiments, it is required to take dispersion and absorption of the $\mathrm{THz}$ pulse into account, which can be done in FDTD by the auxiliary differential equation (ADE), recursive convolution, or Z-transform methods [11]. We have found the most versatile, accurate, and computationally efficient method to be the ADE method [16]. This method is based on solving the equations of motions of charge carriers simultaneously with the solving of the Maxwell equations.

The numerical problem can be reduced considerably by assuming homogeneous transverse excitation of sample and a Rayleigh length of the largest wavelength components of the THz pulse much larger than the thickness of the photoexcited region. This can easily be obtained experimentally for thin samples. This consideration reduces the problem from three to one spatial dimensions. Furthermore, the THz pulse is typically linearly polarized; hence, only one electric and one magnetic field component are necessary. Under these assumptions, the relevant Maxwell equations reduce to

$$
\begin{gathered}
-\frac{\partial E}{\partial z}=\mu_{0} \frac{\partial H}{\partial t}, \\
-\frac{\partial H}{\partial z}=\epsilon_{0} \epsilon_{\infty} \frac{\partial E}{\partial t}+\frac{\partial P_{\Omega}}{\partial t},
\end{gathered}
$$

where $E$ is the electric field, $H$ is the perpendicular component of the magnetic field, $\mu_{0}$ and $\epsilon_{0}$ are the vacuum permeability and vacuum permittivity, and $\epsilon_{\infty}$ is the relative permittivity of the nonexcited sample at $\mathrm{THz}$ frequencies. $P_{\Omega}$ is the induced polarization density that deals with dispersion and absorption due to the photoexcited carriers. $P_{\Omega}$ is governed by

$$
\frac{\partial P_{\Omega}}{\partial t}=-e \sum_{p}\left(x_{p} \frac{\partial N_{p}}{\partial t}+N_{p} \frac{\partial x_{p}}{\partial t}\right),
$$

where $N_{p}$ is the density of a certain type of carrier with index $p, x_{p}$ is the carrier displacement, and $e$ is the electronic charge. The crux of our model is how these dynamic quantities are modeled.

The Lorentz and Drude models of material response are widely used to describe the $\mathrm{THz}$ frequency response of excited molecules and charge carriers [18]. These models can be expressed as the classical, damped, and driven masson-a-spring-like oscillator through the equation of motion:

$$
\frac{\partial^{2} x_{p}(t)}{\partial t^{2}}+\gamma_{p}(t) \frac{\partial x_{p}(t)}{\partial t}+\omega_{0 p}(t)^{2} x_{p}(t)=-\frac{e}{m_{p}^{*}} E(t),
$$

where $\gamma_{p}$ is the momentum scattering rate, $\omega_{0 p}$ is the resonance frequency of the Lorentz model, and $m_{p}^{*}$ is the effective mass of the carrier. In the ADE method, this equation is solved using CDA. Because it is solved in the time-domain, the parameters $\omega_{0}, \gamma$, and $m^{*}$ are allowed to be time dependent and nonlinear terms can be added. This adds a high degree of flexibility in the model. A measured transient response can be factorized such that it is due to the evolution of populations of more than one kind of carriers, e.g., free and localized electrons or phonon population depending on electron-phonon scattering rates.

The temporal evolution of the population density of the photoexcited carriers in our model is assumed to be determined by optical pumping, bulk and surface recombination, diffusion, and relaxation processes. This amounts to

$$
\frac{\partial N_{p}}{\partial t}=y_{p} I-\gamma_{p}^{b} N_{p}+\frac{\partial}{\partial z}\left\{D_{p} \frac{\partial N_{p}}{\partial z}\right\}+\sum_{q \neq p}^{P}\left(\gamma_{p q} N_{q}-\gamma_{q p} N_{p}\right),
$$

where $y_{p}$ is the absorption yield, $I$ is the excitation function given below, $\gamma_{p}^{b}$ is the bulk recombination rate, $D_{p}$ is the diffusion coefficient, and $\gamma_{p q}$ and $\gamma_{q p}$ are the recombination rates between the types of carriers. The summation notation means summing $q$ from 1 to $P$ except $p$. Note that every parameter can depend on $z$ and can be chosen to be dependent on time or another simulated quantity. The frequency of the optical pump is much higher than the plasma frequency of the excited carriers; hence, it is sufficient to propagate the intensity envelope of the pump as an excitation function

$$
\begin{aligned}
I(z, t)= & \frac{N_{i}}{\delta_{\lambda_{p}} \sqrt{\pi} \Delta t_{p}} \exp \left\{-\frac{z}{\delta_{\lambda_{p}}}\right\} \\
& \times \exp \left\{-\frac{\left(z-c_{0} / n_{g}\left(t-t_{p p}\right)\right)^{2}}{\left(c_{0} \Delta t_{p} / n_{g}\right)^{2}}\right\},
\end{aligned}
$$

where $N_{i}$ is the initial density given by $2 \pi F \lambda_{p}(1-R) /\left(\hbar c_{0} \delta_{\lambda_{p}}\right)$, $F$ is the pump fluence, $\delta_{\lambda_{p}}$ is the absorption depth, $\hbar / 2 \pi$ is the Planck constant, $\Delta t_{p}$ is the Gaussian pump pulse width, $n_{g}$ is the group index of the optical pulse, and $t_{p p}$ is the pump-probe delay time.

Surface recombination is modeled through the boundary conditions of the diffusion term, as explained in detail later. We have chosen to model the relaxation processes between different types of carriers by use of rate equations, which have been shown to explain ultrafast measurements of carrier dynamics quite well [19].

\section{A. Definitions and Stability}

The geometry of the numerical simulation is illustrated in Fig. 2. The CDA applied to a spatial derivative in position $i$ at time $n$ using a half-step CDA is defined as

$$
\left.\frac{\partial E}{\partial z}\right|_{i} ^{n} \approx \frac{E_{i+1 / 2}^{n}-E_{i-1 / 2}^{n}}{h}
$$

where $z=i h, h$ is the spatial step size, time $t=n \delta t$, and $\delta t$ is the time step. This implies the Yee grid, where $E$ and $H$ are 


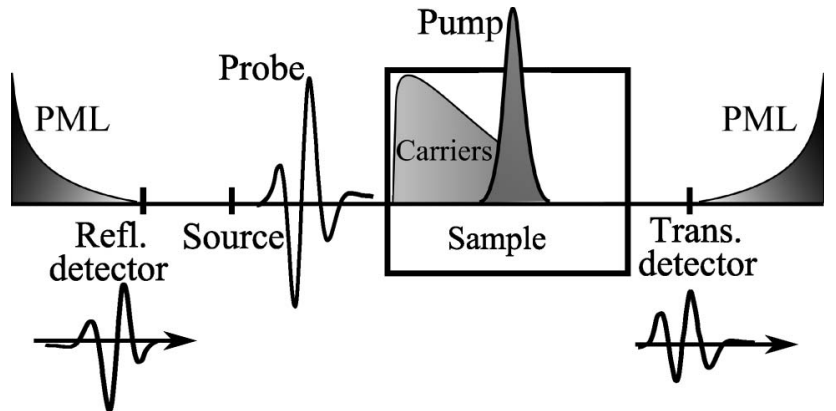

Fig. 2. Illustration of the numerical setup. A source injects the $\mathrm{THz}$ probe pulse, a pump excites carriers in the sample, and detectors measure reflection and transmission. PML are the absorbing perfectly matched layer boundary conditions.

displaced by one-half and staggered, and the time-step is the so-called leapfrog. Other simulated quantities are linked to the position of $E_{i}$. Numerically, this means that spatial arrays are stored during simulations, and for each time step they are updated one after another. The step sizes are related by the Courant number:

$$
Q=c_{0} \delta t / h \leq 1,
$$

where $c_{0}$ is the speed of light, and an interpretation of $Q$ is that it is not possible to propagate a certain distance $h$ per unit time $\delta t$ with a velocity faster than $c_{0}$. Similar relations can be put forward for diffusion coefficient $D$ and surface recombination velocity $s$ :

$$
\frac{2 \delta t D}{h^{2}}<1, \quad \frac{\delta t s}{2 h}<1 .
$$

It was found that the FDTD method is robust as long as spatial or temporal variations, such as the photoexcited region or temporal width of the pump pulse, are discretized with more than 20 numerical points.

\section{B. FDTD Formulation}

Deriving Eqs. (5)-(9) in the FDTD formalism is straightforward, and the result is presented in the following. Furthermore, the applied boundary conditions and a corrective term to the displacement are explained. The onset is applying CDA to the Faraday law in Eq. (5) in the point $\left.\right|_{i+1 / 2} ^{n+1}$ and to Ampere's law in Eq. (ㅁ) in the point $\left.\right|_{i} ^{n+1 / 2}$. To solve the latter, knowledge of the dynamics of the induced polarization density in Eq. (7) is required:

$$
\left.\frac{\partial P_{\Omega}}{\partial t}\right|_{i} ^{n+1 / 2}=-\frac{e}{\delta t}\left(N_{p i}^{n+1} x_{p i}^{n+1}-N_{p i}^{n} x_{p i}^{n}\right) .
$$

Thus, to solve this, the dynamics of the density and the displacement must be solved for each time step. Applying the CDA to Eq. (ㅇ) and solving for $x_{p i}^{n+1}$ leads to

$$
x_{p i}^{n+1}=\alpha_{p i}^{n} x_{p i}^{n}+\beta_{p i}^{n} x_{p i}^{n-1}-\theta_{p i}^{n} E_{i}^{n},
$$

with the definitions

$$
\begin{gathered}
\alpha_{p i}^{n}=\frac{4-2\left(\omega_{0 p i}^{n}\right)^{2} \delta t^{2}}{\gamma_{p i}^{n} \delta t+2}, \\
\beta_{p i}^{n}=\frac{\gamma_{p i}^{n} \delta t-2}{\gamma_{p i}^{n} \delta t+2},
\end{gathered}
$$

$$
\theta_{p i}^{n}=\frac{2 e \delta t^{2}}{m_{p}^{*}\left(\gamma_{p i}^{n} \delta t+2\right)} .
$$

It is cumbersome to solve the population dynamics in Eq. (9) using the CDA for the time step; hence, we have chosen to use a simpler forward difference, and the updating scheme of the dynamics now can be written

$$
\begin{gathered}
N_{p i}^{n+1}=N_{p i}^{n}+\left.\delta t \frac{\partial N_{p}}{\partial t}\right|_{i} ^{n} \\
=\delta t\left\{N_{p i}^{n}\left(\frac{1}{\delta t}-\gamma_{p i}^{b}-\frac{2 D_{p i}^{n}}{h^{2}}\right)\right. \\
+\sum_{q \neq p}\left[\gamma_{p q} N_{q i}^{n}-\gamma_{q p} N_{p i}^{n}\right]+y_{p} I_{i}^{n} \\
\left.+\frac{D_{p i}^{n}}{h^{2}}\left(N_{p i+1}^{n}+N_{p i-1}^{n}\right)\right\} .
\end{gathered}
$$

The excitation function in Eq. (10) is discretized as

$$
\begin{aligned}
I_{i}^{n}= & \frac{2 \pi F \lambda_{p}(1-R)}{\hbar c_{0} \delta_{\lambda_{p}}^{2} \sqrt{\pi} \Delta t_{p}} \exp \left\{-\frac{i h-z_{0}}{\delta_{\lambda_{p}}}\right\} \\
& \times \exp \left\{-\frac{\left[i h-z_{0}-c_{0} / n_{g}\left(n \delta t+\tau_{\text {pump }}-\tau_{\text {probe }}\right)\right]^{2}}{\left(c_{0} \Delta t_{p} / n_{g}\right)^{2}}\right\},
\end{aligned}
$$

where $z_{0}$ is the surface position of the sample, $\tau_{\text {pump }}$ is the pump delay time, and $\tau_{\text {probe }}$ is the probe delay time. At the sample surface there is no diffusion, but instead, surface recombination and such a boundary condition for the left boundary $z_{0}=i_{L} h$ results in

$$
\begin{aligned}
N_{p i_{L}}^{n+1}= & \delta t\left\{N_{p i_{L}}^{n}\left(\frac{1}{\delta t}-\frac{s_{p}^{n}}{h}-\gamma_{p i_{L}}^{b}-\frac{D_{p i_{L}}^{n}}{h^{2}}\right)\right. \\
& \left.+\sum_{q \neq p}\left[\gamma_{p q} N_{q i_{L}}^{n}-\gamma_{q p} N_{p i_{L}}^{n}\right]+y_{p} I_{i_{L}}^{n}+\frac{D_{p i_{L}}^{n}}{h^{2}} N_{p i_{L}+1}^{n}\right\},
\end{aligned}
$$

where $s_{p}^{n}$ is the surface recombination velocity. The remaining task is to solve the Maxwell equations. In order to include the electric and magnetic field boundary conditions and source terms, they are generalized to

$$
\begin{gathered}
-\frac{\partial E}{\partial z}=\mu_{0} \frac{\partial H}{\partial t}+\sigma^{*} H+j_{H}, \\
-\frac{\partial H}{\partial z}=\epsilon_{0} \epsilon_{\infty} \frac{\partial E}{\partial t}+\epsilon_{0} \frac{\partial P_{\Omega}}{\partial t}+\sigma E+j_{E},
\end{gathered}
$$

where $\sigma$ and $\sigma^{*}$ are the electric and (numerical) magnetic conductivity and $j_{E}$ and $j_{H}$ are the source terms. Absorbing boundaries are necessary, and they are modeled by perfectly matched layers (PMLs), which have the property of being reflectionless [16]. This is obtained by impedance matching; $\sigma^{*} \epsilon_{0} \epsilon_{\infty}=\sigma \mu_{0}$. To avoid numerical reflections, a polynomial dependence of the absorption is chosen and a PML parameter $A_{i}$ is defined as

$$
A_{i}=\frac{\sigma_{i} \delta t}{2 \epsilon_{i}}=\frac{\sigma_{i}^{*} \delta t}{2 \mu_{i}}=A_{\max }\left(\frac{i}{A_{h}}\right)^{A_{m}} .
$$

To ensure sufficiently absorbing and reflectionless boundaries, the parameters must be optimized for the simulated THz probe pulse. Typical parameters are $A_{h}=20, A_{\max }=$ 0.2 , and $A_{m}=1.5$. To ease the notation and implementation, the magnetic field is scaled and virtually shifted: 


$$
\tilde{H}_{i+1 / 2}^{n+1 / 2} \rightarrow \sqrt{\epsilon_{0} / \mu_{0}} H_{i}^{n}
$$

The magnetic field is evolved by

$$
H_{i}^{n+1}=\frac{1-A_{i+1 / 2}}{1+A_{i+1 / 2}}\left\{H_{i}^{n}-\frac{Q\left(E_{i+1}^{n+1}-E_{i}^{n+1}\right)}{1-A_{i+1 / 2}}\right\}+Q j_{E i}^{n},
$$

where $Q j_{E i}^{n}$ is the source term resulting in the right propagating input. Finally, the electric field is updated as

$$
\begin{aligned}
E_{i}^{n+1}= & \frac{1-A_{i}}{1+A_{i}}\left\{E_{i}^{n}-\frac{Q}{\epsilon_{\infty i}} \frac{H_{i}^{n}-H_{i-1}^{n}}{1-A_{i}}\right\} \\
& +\frac{e}{\epsilon_{0} \epsilon_{\infty i}} \sum_{p}\left(N_{p i}^{n+1} x_{p i}^{n+1}-N_{p i}^{n} x_{p i}^{n}\right)+j_{E i}^{n},
\end{aligned}
$$

where it is assumed that $N_{i}=0$ for $A_{i} \neq 0$, meaning the PML boundaries are not photoexcited.

A corrective term must be implemented after updating the carrier densities but before calculating new displacements. It relies on causality; so, for example, a carrier cannot be displaced before it has been excited. Consider the average displacement after an photoexcitation event $\tilde{x}_{p i}^{n}$ :

$$
\tilde{x}_{p i}^{n}=\frac{N_{p i}^{n} x_{p i}^{n}+0 \cdot I_{i}^{n} \delta t}{N_{p i}^{n}+I_{i}^{n} \delta t} .
$$

This is equivalent to the fact that the newly photoexcited carriers are at rest just after the excitation event. By extending this principle for all the dynamic processes, a generalized expression is

$$
\tilde{x}_{p i}^{n}=\frac{N_{p i}^{n} x_{p i}^{n}+\sum_{\phi} \Delta N_{\phi i}^{n} x_{\phi i}^{n}}{N_{p i}^{n+1}},
$$

where $\Delta N_{\phi i}^{n}$ and $x_{\phi i}^{n}$ are listed in Table $\underline{1}$. Similar arguments can be put forward for the momentum of a carrier after an event:

$$
\tilde{x}_{p i}^{n-1}=\frac{x_{p i}^{n-1}+\sum_{\phi} \Delta N_{\phi i}^{n}\left\{x_{\phi i}^{n}\left(1-\frac{m_{\phi}^{*}}{m_{p}^{*}}\right)+\frac{m_{\phi}^{*}}{m_{p}^{*}} x_{\phi i}^{n-1}\right\}}{N_{p i}^{n+1}} .
$$

\section{EXPERIMENTAL METHODS}

To validate the FDTD method, TRTS experiments have been carried out. The experimental method and setup have been described elsewhere [7,20]. In short, they consist of a

Table 1. Corrective Terms to Be Used in Eqs. (30) and (31) ${ }^{a}$

\begin{tabular}{lcc}
\hline Process & $\Delta N_{\phi i}^{n} / \delta t$ & $x_{\phi i}^{n}$ \\
\hline Excitation & $y_{p} I_{i}^{n}$ & 0 \\
Bulk recombination & $-\gamma_{p i}^{b} N_{p i}^{n}$ & $x_{p i}^{n}$ \\
Surface recombination & $-s_{p}^{n} N_{p i}^{n} / h$ & $x_{p i}^{n}$ \\
Diffusion out & $-2 D_{p i}^{n} N_{p i}^{n} / h^{2}$ & $x_{p i}^{n}$ \\
Diffusion in & $D_{p i}^{n} N_{p i \pm 1}^{n} / h^{2}$ & $x_{p i \pm 1}^{n}$ \\
Coupling out & $-\sum_{q \neq p} \gamma_{q p} N_{p i}^{n}$ & $x_{p i}^{n}$ \\
Coupling in $\left(m_{\phi}^{*}=m_{q}^{*}\right)$ & $\sum_{q \neq p} \gamma_{p q} N_{q i}^{n}$ & $x_{q i}^{n}$ \\
\hline
\end{tabular}

${ }^{a}$ In every process, $m_{\phi}^{*}=m_{p}^{*}$, except for coupling in, which has $m_{\phi}^{*}=m_{q}^{*}$.
Ti:sapphire regenerative fs laser amplifier delivering $45 \mathrm{fs}$, millijoule pulses at a $1 \mathrm{kHz}$ repetition rate.

The pulses are split into pump, probe, and sampling parts, which are delayed relative to each other by mechanical delay stages. The THz pulse is generated using a $515 \mu \mathrm{m}\langle 110\rangle \mathrm{ZnTe}$ crystal, and it is collimated by off-axis parabolic Al-coated mirrors of $1^{\prime \prime}$ and $3^{\prime \prime}$ focal length. The THz pulse is then focused to a diffraction limit spot of $\sim 1 \mathrm{~mm}$ at the sample. The pump beam spot size on the sample is at least three times larger than the THz pulse spot. The transmitted THz pulse is recollimated and focused by a $3^{\prime \prime}$ focal length mirror onto a $515 \mu \mathrm{m}\langle 110\rangle \mathrm{ZnTe}$ crystal, where it copropagates with the sampling pulse for electro-optic detection. The reference and differential waveforms are measured simultaneously to reduce acquisition time and changes in the experimental conditions during measurements [20]. The measurements are carried out by fixing the pump and sampling pulses for a given $t_{p}$, and the probe pulse is scanned. This means that the reconstructed 2D map is measured.

The sample is $\langle 100\rangle$ GaAs with thickness of $500 \mu \mathrm{m}$ mounted on a $2 \mathrm{~mm}$ aperture.

For completeness, the equations used in the analysis and considerations on the Fourier transform are given. Using the property in Eq. (3), the transient transmission function can be found as

$$
T\left(\Omega, t_{p}\right)=\frac{E_{\mathrm{pump}}\left(\Omega, t_{p}\right)}{E_{\mathrm{ref}}\left(\Omega, t_{p}\right)}=\left|T\left(\Omega, t_{p}\right)\right| e^{i \Phi\left(\Omega, t_{p}\right)},
$$

where $|T|$ is the transmission amplitude and $\Phi$ is the phase change. The transient, complex conductivity $\sigma=\sigma_{1}+i \sigma_{2}$ can, in this work, be found using the thin-film equation [7]:

$$
\begin{gathered}
\sigma_{1}(\Omega)=\frac{n(\Omega)+1}{Z_{0} \delta_{\lambda_{p}}}\left(\frac{\cos \Phi}{|T|}-1\right), \\
\sigma_{2}(\Omega)=-\frac{n(\Omega)+1}{Z_{0} \delta_{\lambda_{p}}} \frac{\sin \Phi}{|T|},
\end{gathered}
$$

where $n(\Omega)$ is the static THz refractive index, which for GaAs is $3.61 ; Z_{0}$ the vacuum impedance; and $\delta_{\lambda_{p}}$ is the absorption depth, which is assumed to be $700 \mathrm{~nm}$.

As described in Section 2, the THz probe pulses experience modulation due to the experimental setup and detection, which can be described by the response functions. The detector response function is found in [14], under the described approximations, to be

$$
\begin{gathered}
f_{\text {det }}(\Omega) \approx \chi^{(2)}\left(\omega_{0}, \Omega\right) \frac{e^{i \Delta k\left(\omega_{0}, \Omega\right) d}-1}{i \Delta k\left(\omega_{0}, \Omega\right)} \frac{2}{1+n(\Omega)} \\
\times \int A^{*}(\omega) A(\omega-\Omega) \mathrm{d} \omega, \\
\Delta k\left(\omega_{0}, \Omega\right) \approx \frac{\Omega\left\{n_{g}\left(\omega_{0}\right)-n(\Omega)\right\}}{c_{0}},
\end{gathered}
$$

where $\chi^{(2)}$ is the effective second-order susceptibility at the optical $\omega_{0}$ and THz $\Omega$ frequency, $\Delta k$ is the complex phase mismatch parameter, $d$ is the crystal thickness, $n_{g}$ is the optical group index, $n(\Omega)$ is the THz refractive index, and the integral over $A^{*} A$ is the autocorrelation of the optical field. 
The aperture in the setup is due to focusing of both the $\mathrm{THz}$ and the sampling pulse at the detector, by which the detection sensitivity of the low-frequency components are reduced. An appropriate response function is derived in [15], which at the focus is given by

$$
f_{\text {apr }}(\Omega)=\operatorname{erf}^{2}\left(\frac{\Omega R}{2 c_{0}}\right),
$$

where $R$ is the beam waist of the sampling pulse. The response functions are shown in Fig. 3 .

To reduce measuring time and still obtain high-resolution data (information density) in the frequency domain, we chose to only capture the exact probe pulse, see Fig. $\underline{4}$, and then zero pad to decrease the frequency spacing given by the reciprocal of the temporal window, $\delta f=1 / T$. In the measurements, 200 points are distributed over $4 \mathrm{ps}$ and zero padding is carried out to extend the temporal window to 1024 points. It has been ensured that the electric field at the edges of the temporal domain rapidly goes to zero. As the electric field is very low at times before and after the probe pulse, it can be argued that this zero padding replaces measurement noise before and after the pulse, which would be time consuming and give no additional information.

The simulation yields a 2D map of $E_{\text {pump }}\left(t, t_{p p}\right)$, and to reconstruct $E_{\text {pump }}\left(t, t_{p}\right)$ to enable the Fourier transform, a linear interpolation scheme with $t_{p p}=t+t_{p}$ is applied.

In the simulation shown in the next section, the following numerical parameters are used. A single term of the Drude type with $\gamma=2 \pi \cdot 1.3 \mathrm{THz}$ (momentum scattering time $\tau=122 \mathrm{fs}$ ), $m^{*}=0.067, s=10^{6} \mathrm{~cm} / \mathrm{s}, D=21 \mathrm{~cm}^{2} / \mathrm{s}$, and $\epsilon_{\infty}=13$. The input pulse is the deconvoluted reference pulse from the experiment. The pump is simulated with $n_{g}=4, \Delta t_{p}=50 \mathrm{fs}$, $F=8 \mu \mathrm{J} / \mathrm{cm}^{2}, \lambda_{p}=800 \mathrm{~nm}, \delta_{\lambda_{p}}=700 \mathrm{~nm}, R=0.33$. Finally, the number of numerical spatial and temporal steps are $N_{h}=2^{9}$ and $N_{t}=2^{16}$, respectively.

\section{DATA ANALYSIS USING THE FDTD METHOD}

In the following, analysis of TRTS data of GaAs pumped at $800 \mathrm{~nm}$ at a pump fluence of $7 \mu \mathrm{J} / \mathrm{cm}^{2} /$ pulse is carried out

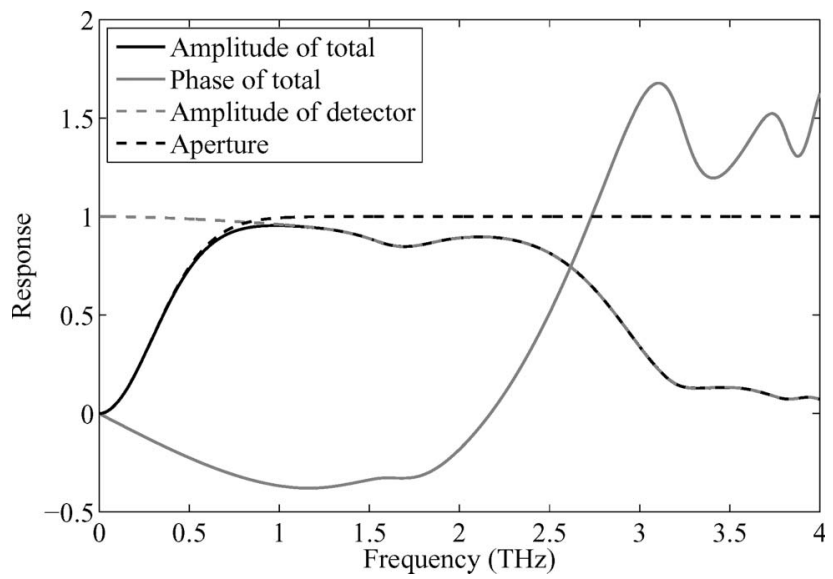

Fig. 3. Amplitude and phase of the total response function $\left|f_{\text {det }} f_{\text {apr }}\right|$ and $\operatorname{atan}\left(\mathfrak{J}\left\{f_{\text {det }} f_{\text {apr }}\right\} / \mathfrak{R}\left\{f_{\text {det }} f_{\text {apr }}\right\}\right)$, respectively. The amplitude of the individual response functions of the aperture and detector using Eqs. (35) and (37) is also shown. The THz refractive index of ZnTe is measured, and the nonlinearity is modeled as $\chi^{(2)} \propto n(\Omega)^{2}-1$ [14]. The aperture radius or sampling beam waist is $200 \mu \mathrm{m}$.

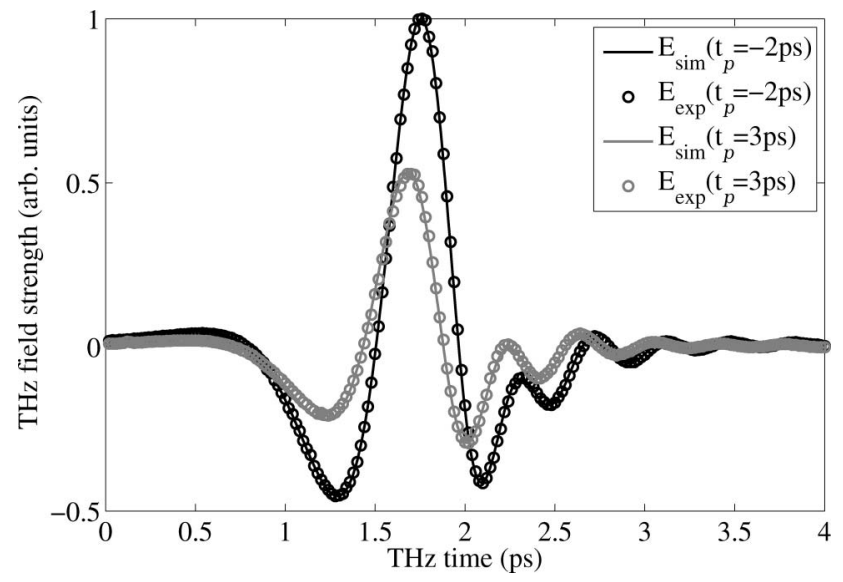

Fig. 4. Measured and simulated electric field of the THz probe pulses before $\left(t_{p}=-2 \mathrm{ps}\right)$ and after $\left(t_{p}=3 \mathrm{ps}\right)$ optical pumping. The GaAs sample is excited with $800 \mathrm{~nm}, 7 \mu \mathrm{J} / \mathrm{cm}^{2}, \sim 45$ fs pulses.

using FDTD simulation results. GaAs photoexcited at these parameters will result in excitation densities of $<10^{18} \mathrm{~cm}^{-3}$, and due to the low excess energy of $\sim 130 \mathrm{meV}$ only the central $\Gamma$ valley is expected to be populated. This direct population of a single valley result in an ultrafast carrier response time that is given by the duration of the pump pulse of $\sim 45 \mathrm{fs}$ [1].

The focus in the analysis will be on early $2 \mathrm{D}$ data, which cannot be analyzed under the quasistatic approximation. Furthermore, the probe pulse attenuation is $~ 50 \%$ at the used pump fluence; hence, the linearized analysis cannot be applied. Moreover, the 2D Fourier transform method is challenged due to the very slow carrier recombination times of several nanoseconds.

However, the FDTD method can very well describe the onedimensional (1D) pump experiment due to inclusion of the carrier diffusion and rate equation capabilities. 1D probe or static experiments can be analyzed as well by propagating the THz probe in a dispersive material. Finally, it can be very instructive to study the simulation in "real time" to visualize the involved dynamics of the propagating $\mathrm{THz}$ pulse and carriers.

In Fig. $\underline{4}$, the time-domain waveforms measured before and after the pumping event are shown together with the simulation results. The high degree of similarity shows that the method at the quantitative level can simulate the probe pulse propagation in a TRTS experiment.

Two approaches to the complete analysis of the TRTS experiment using the FDTD method are described in the following:

1. Comparing the simulated conductivity with the experimentally obtained conductivity where the response functions have been deconvoluted.

2. Convoluting the simulation with the response functions before extracting the conductivity and comparing this with the experimental conductivity.

\section{A. Deconvolution Experiment Results}

As a deconvolution is the inverse of a convolution, it consists of dividing $\tilde{E}(\Omega)$ by the response function $f(\Omega)$. However, as the response function goes to zero for high frequencies (all information is lost) the deconvoluted field $E(\Omega)$ will have unphysical large amplitudes at high frequencies. That is, 


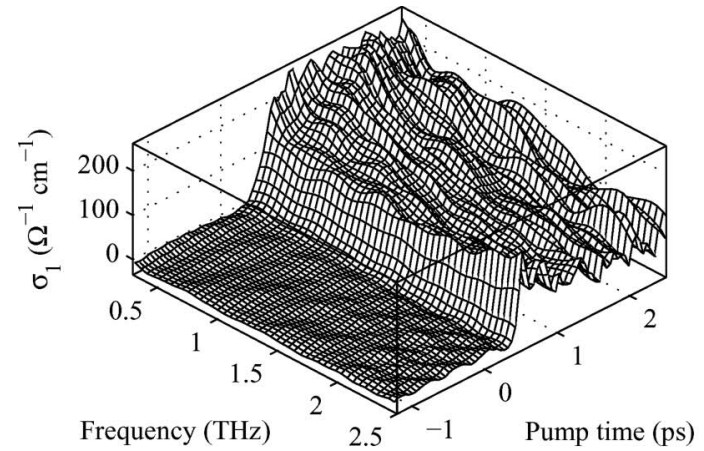

(a) Deconvoluted experiment: $\sigma_{1}$

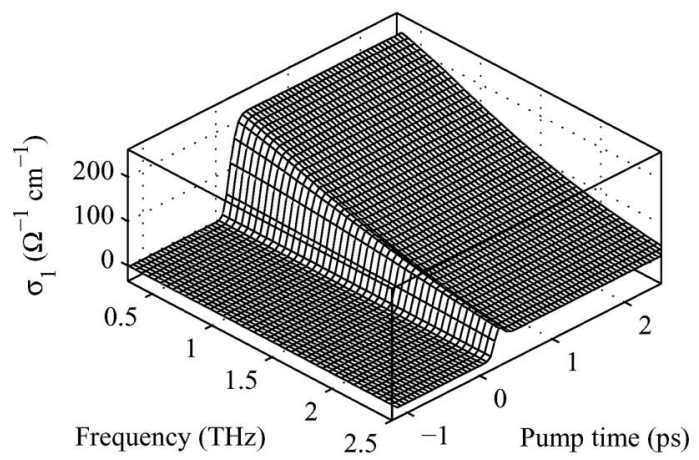

(c) Simulation: $\sigma_{1}$

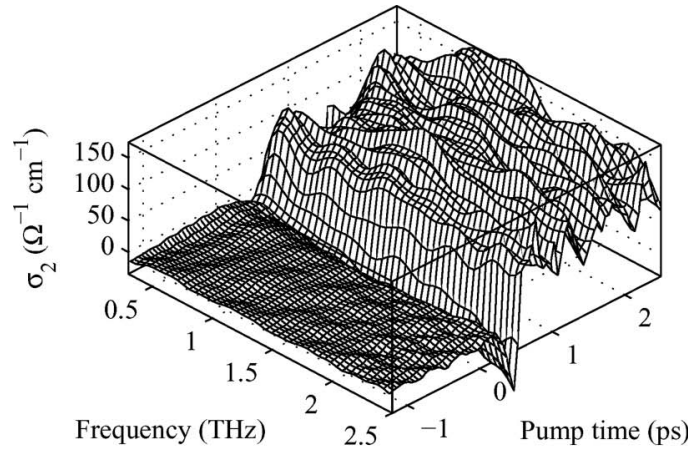

(b) Deconvoluted experiment: $\sigma_{2}$

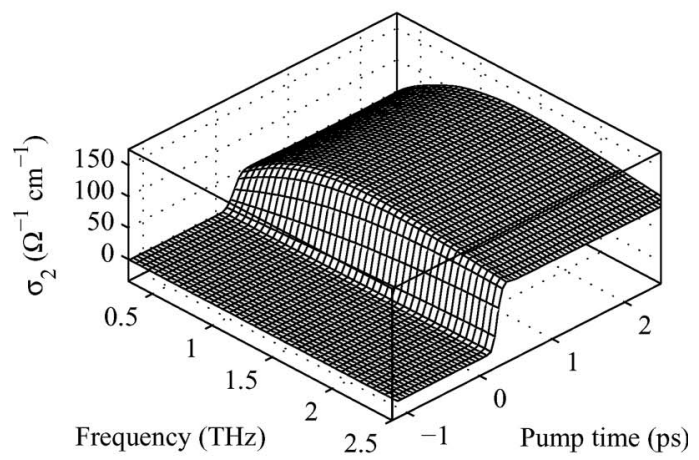

(d) Simulation: $\sigma_{2}$

Fig. 5. Real $\left(\sigma_{1}\right)$ and imaginary $\left(\sigma_{2}\right)$ transient conductivity of the experiment and the simulation. Before extracting the experimental conductivity in (a) and (b), the response functions have been deconvoluted in order to be directly comparable to the simulation results in (c) and (d). The GaAs sample is excited with $800 \mathrm{~nm}, 7 \mu \mathrm{J} / \mathrm{cm}^{2}, \sim 45$ fs pulses. The simulation parameters are given in Section $\underline{4}$.

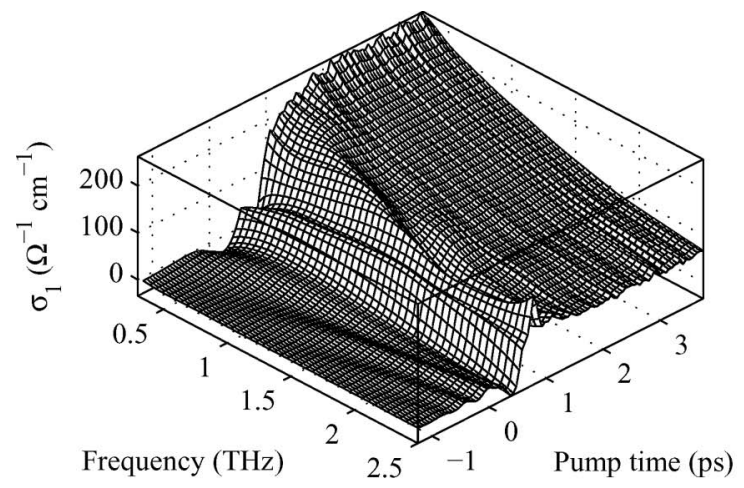

(a) Experiment

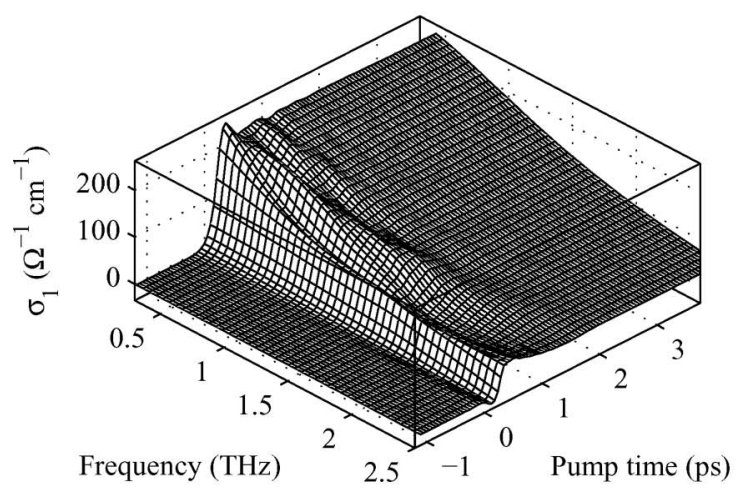

(c) Detector

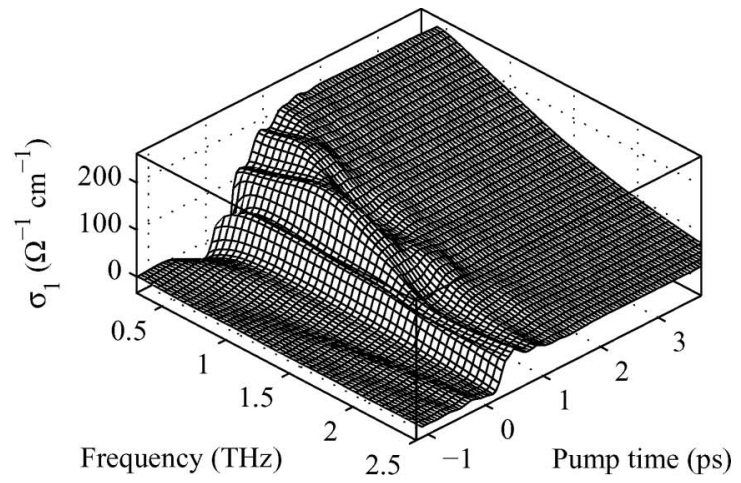

(b) Detector and aperture

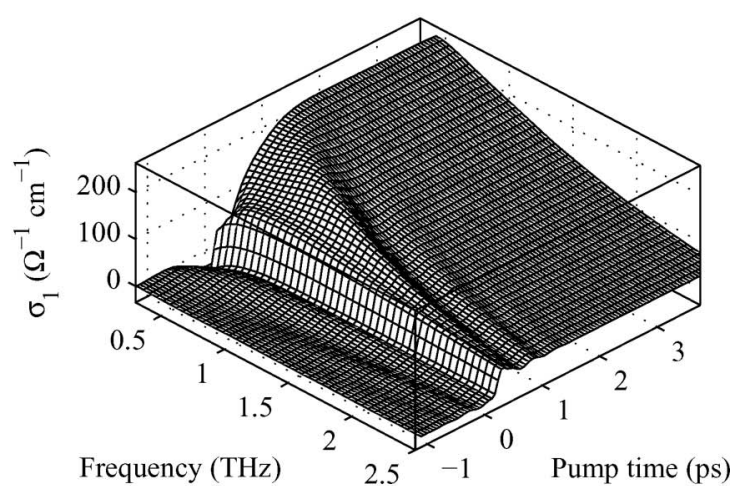

(d) Aperture

Fig. 6. Real part of conductivity $\sigma_{1}$ for (a) experiment, (b) simulation convoluted with the detector and aperture response functions before reconstruction and Fourier transformation, (c) as (b) convoluted with the detector function only, and (d) as (b) convoluted with the aperture function only. The GaAs sample is excited with $800 \mathrm{~nm}, 7 \mu \mathrm{J} / \mathrm{cm}^{2}, \sim 45 \mathrm{fs}$ pulses. The simulation parameters are given in Section 4 . 


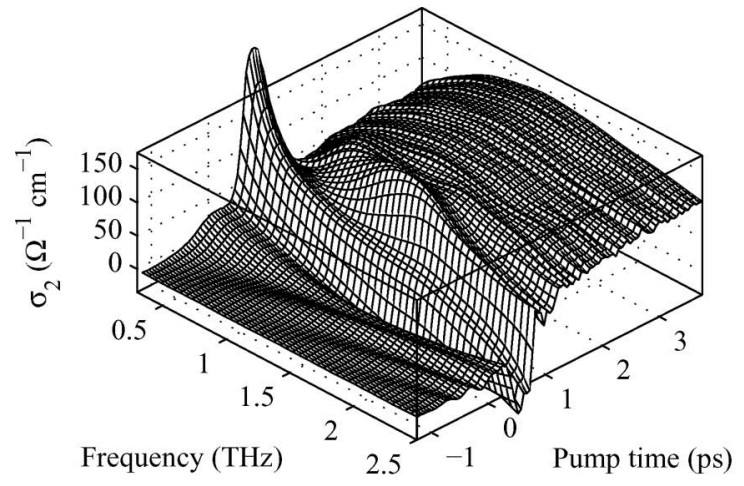

(a) Experiment

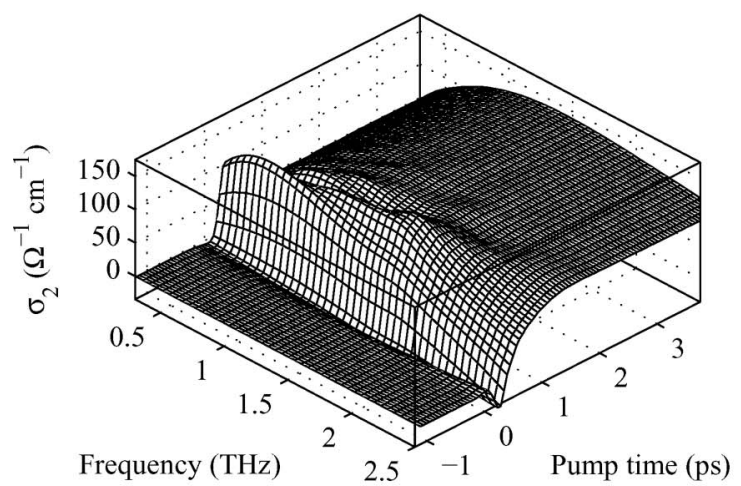

(c) Detector

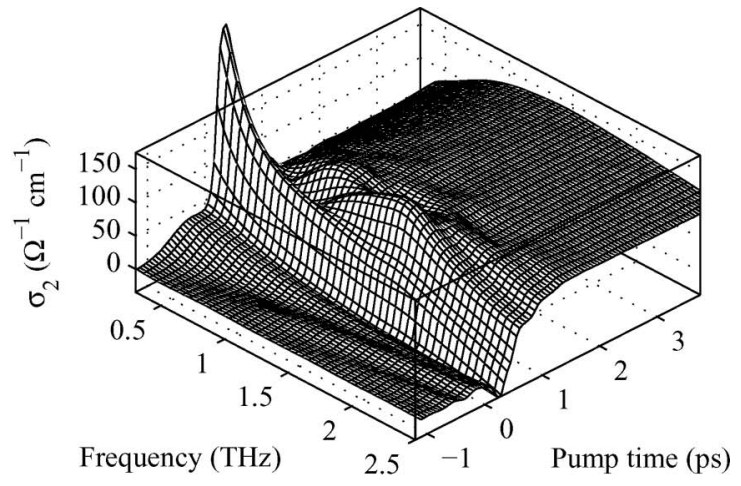

(b) Detector and aperture

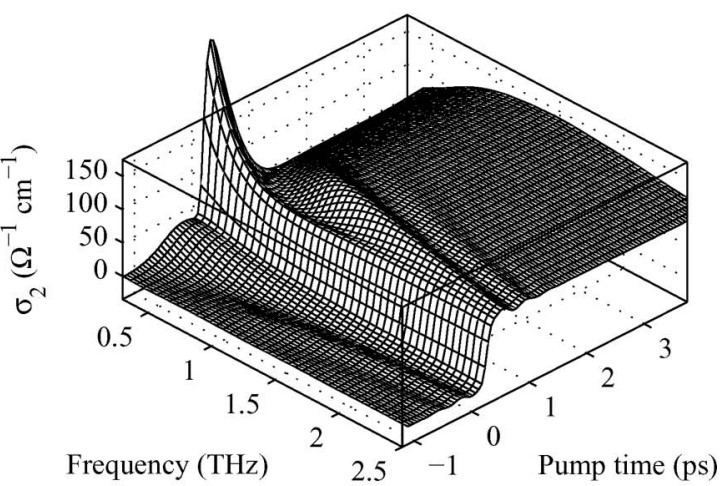

(d) Aperture

Fig. 7. Imaginary part of conductivity $\sigma_{2}$ for (a) experiment, (b) simulation convoluted with the detector and aperture response functions before reconstruction and Fourier transformation, (c) as (b) convoluted with the detector function only, and (d) as (b) convoluted with the aperture function only. The GaAs sample is excited with $800 \mathrm{~nm}, 7 \mu \mathrm{J} / \mathrm{cm}^{2}, \sim 45 \mathrm{fs}$ pulses. The simulation parameters are given in Section $\underline{4}$.

deconvolution leads to amplification of small amplitude signals with a high level of noise. To be able to deconvolute the data, cut-off frequencies are chosen at the frequencies where the response is $10 \%$, which corresponds to 0.13 and $3.7 \mathrm{THz}$.

Figures 5(a) and 5(b) show the complex conductivity extracted by Eqs. (33) and (34) from the experimental data deconvoluted with the response functions in Fig. 3 using the method described above. The simulated complex conductivity is shown in Figs. 5 (c) and $5(\mathrm{~d})$. It can be seen that the experimental data become noisy due to the deconvolution process. Therefore, any parameter extracted from the data will have a high uncertainty, and it seems that this approach is not preferable.

\section{B. Convoluting Simulation Results}

Alternatively, instead of processing the experimental data with noise generating procedures, the simulation can be convoluted with the response functions to mimic the experimental results accurately. The procedure is that after simulation, $E_{\text {pump }}\left(t, t_{p p}\right)$ is Fourier transformed to $E_{\text {pump }}\left(\Omega, t_{p p}\right)$ to do the convolution $f(\Omega) E_{\text {pump }}\left(\Omega, t_{p p}\right)=\tilde{E}_{\text {pump }}\left(\Omega, t_{p q}\right)$, then inverse Fourier transformed back to time domain $E_{\text {pump }}\left(t, t_{p p}\right)$. It is then reconstructed to $\tilde{E}_{\text {pump }}\left(t, t_{p}\right)$ and Fourier transformed to $\tilde{E}_{\text {pump }}\left(\Omega, t_{p}\right)$, and finally the conductivity $\sigma\left(t, t_{p}\right)$ is extracted.

The results are shown in Figs. $\underline{6}$ and $\underline{7}$, where the real and imaginary part of the conductivities are shown, respectively. First, by comparing the experimental conductivity in Figs. 6(a) and 7(a) with the simulated conductivity in Figs. 5(c) and 5(d), it is clear that artifacts emerge around the pumping event. These artifacts can hardly be described by any carrier physics. As an example, the waves before the pumping event appear to violate causality. After $t_{p}=2 \mathrm{ps}$, no artifacts are present and the conductivity can be described by the Drude type conductivity and the quasistatic approximation holds.

Second, by convoluting the simulations with the response functions, the curious artifacts can be accurately reproduced, as can be seen by comparing Figs. 6(a) and 6(b), and the same is seen by comparing Figs. 7(a) and 7(b). Because of the large degree of resemblance, it can be concluded that the simulated physics and the response functions describe the experimental system well. Hence, by this method we have shown a way to determine the ultrafast carrier dynamics occurring after optical pumping of the sample and, more importantly, that the temporal resolution is given by the duration of the optical pump pulse.

Finally, in Figs. 6(c), 6(d), 7(c), and 7(d), the conductivities calculated only by convoluting with one of the response functions are shown. They show the constituents of the artifacts, and we see that the detector and the aperture contribute very differently to these artifacts. We can conclude that each of these response functions must be taken into account to analyze the data.

\section{CONCLUSIONS}

From the analysis of the TRTS experiment, two problems were discussed for early pump-probe delay times. The limit of "early" times is given by the largest characteristic time of the THz pulse, material response, or detector response. For larger delay times, any static Fourier transform method can be applied. If reconstructed $2 \mathrm{D}$ data $E_{\text {pump }}\left(t, t_{p}\right)$ are obtained, 
Fourier transform methods can be used as long as the material response time is larger than the detector response time. Finally, for material responses comparable to or faster than the detector response, (de)convolution of the experimental setup responses is required.

The numerical analysis of a time-resolved $\mathrm{THz}$ spectroscopy experiment using a modified FDTD method has been described and discussed. Utilizing this method, we showed that ultrafast carrier dynamics can be determined with a time resolution smaller than the duration of the $\mathrm{THz}$ probe pulse and only determined by the pump pulse.

The FDTD implementation is versatile, and we plan to use it for analysis of numerous TRTS experiments. The numerical implementation is available online [21].

\section{REFERENCES}

1. J. Shah, Ultrafast Spectroscopy of Semiconductors and Semiconductor Nanostructures (Springer-Verlag, 1999).

2. A. Othonos, "Probing ultrafast carrier and phonon dynamics in semiconductors," J. Appl. Phys. 83, 1789-1830 (1998).

3. G. Gallot, J. Zhang, R. McGowan, T. Jeon, and D. Grischkowsky, "Measurements of the $\mathrm{THz}$ absorption and dispersion of $\mathrm{ZnTe}$ and their relevance to the electro-optic detection of $\mathrm{THz}$ radiation," Appl. Phys. Lett. 74, 3450-3452 (1999).

4. R. Huber, F. Tauser, A. Brodschelm, M. Bichler, G. Abstreiter, and A. Leitenstorfer, "How many-particle interactions develop after ultrafast excitation of an electron-hole plasma," Nature 414, 286-289 (2001).

5. R. A. Kaindl, M. A. Carnahan, D. Hagele, R. Lovenich, and D. S. Chemla, "Ultrafast terahertz probes of transient conducting and insulating phases in an electron-hole gas," Nature 423, 734-738 (2003).

6. C. Schmuttenmaer, "Exploring dynamics in the far-infrared with terahertz spectroscopy," Chem. Rev. 104, 1759-1780 (2004).

7. P. U. Jepsen, D. G. Cooke, and M. Koch, "Terahertz spectroscopy and imaging-modern techniques and applications," Laser Photon. Rev. 5, 124-166 (2011).

8. H. Němec, F. Kadlec, and P. Kužel, "Methodology of an optical pump-terahertz probe experiment: an analytical frequencydomain approach,” J. Chem. Phys. 117, 8454-8466 (2002).
9. M. Beard, G. Turner, and C. Schmuttenmaer, "Transient photoconductivity in GaAs as measured by time-resolved terahertz spectroscopy," Phys. Rev. B 62, 15764-15777 (2000).

10. J. Kindt and C. Schmuttenmaer, "Theory for determination of the low-frequency time-dependent response function in liquids using time-resolved terahertz pulse spectroscopy," J. Chem. Phys. 110, 8589-8596 (1999).

11. M. Beard and C. Schmuttenmaer, "Using the finite-difference time-domain pulse propagation method to simulate timeresolved THz experiments," J. Chem. Phys. 114, 2903-2909 (2001).

12. M. Beard, G. Turner, and C. Schmuttenmaer, "Subpicosecond carrier dynamics in low-temperature grown GaAs as measured by time-resolved terahertz spectroscopy,” J. Appl. Phys. 90, 5915-5923 (2001).

13. H. Němec, F. Kadlec, S. Surendran, P. Kužel, and P. Jungwirth, "Ultrafast far-infrared dynamics probed by terahertz pulses: a frequency domain approach. I. model systems," J. Chem. Phys. 122, 104503 (2005).

14. G. Gallot and D. Grischkowsky, "Electro-optic detection of terahertz radiation,” J. Opt. Soc. Am. B 16, 1204-1212 (1999).

15. D. Côté, J. Sipe, and H. van Driel, "Simple method for calculating the propagation of terahertz radiation in experimental geometries," J. Opt. Soc. Am. B 20, 1374-1385 (2003).

16. A. Taflove and S. Hagness, Computational Electrodynamics: The Finite-Difference Time-Domain Method (Artech, 2005).

17. W. Pernice, F. Payne, and D. Gallagher, "A finite-difference timedomain method for the simulation of gain materials with carrier diffusion in photonic crystals," J. Lightwave Technol. 25, 2306-2314 (2007).

18. M. Dressel and M. Scheffler, "Verifying the Drude response," Ann. Phys. 15, 535-544 (2006).

19. C. Stanton and D. Bailey, "Rate equations for the study of femtosecond intervalley scattering in compound semiconductors," Phys. Rev. B 45, 8369-8377 (1992).

20. K. Iwaszczuk, D. G. Cooke, M. Fujiwara, H. Hashimoto, and P. Jepsen, "Simultaneous reference and differential waveform acquisition in time-resolved terahertz spectroscopy," Opt. Express 17, 21969-21976 (2009)

21. C. Larsen, D. G. Cooke, and P. U. Jepsen, "A numerical implementation in MATLAB," available online at http://www .physics.mcgill.ca/ cooke/ and http://www.terahertz.dk (2011). 